\title{
The Design of a Highly Reliable Robot for Unmediated Museum Interaction
}

\author{
Illah Nourbakhsh, Emily \\ Hamner, Eric Porter, Brian \\ Dunlavey and Ellen Ayoob \\ The Robotics Institute \\ Carnegie Mellon University \\ Pittsburgh, PA, USA
}

\author{
Thomas Hsiu \\ Gogoco LLC \\ Sunnyvale, CA, USA
}

\author{
Mark Lotter and Skip Shelly \\ LotterShelly \\ Pittsburgh, PA, USA
}

\begin{abstract}
Installation of a robot system in a publicly accessible technical museum poses nontrivial problems along three axes. First, the robot must be reliable, both by failing rarely in spite of continuous, daily use and by allowing museum staff to easily return the robot to service. Second, the robot must perform without the need for staff intervention, from system autonomy to energetics enabling full-day operation without battery replacement. Third, the user-end interaction software must be self-explanatory as well as instructional and engaging in order to effectively communicate the learning goals of the exhibit. In this paper we describe the design of such a robot system and share early results regarding its successful deployment at five museums across the United States.
\end{abstract}

Index Terms - Reliability, Morphology, Human-Robot Interaction, Interaction Design

\section{INTRODUCTION}

Critical enabling technologies for long-term, high competence mobile robotics have made significant strides over the past few years. In conjunction with this greatly increased potential for mobile robots to interact intelligently with humans, the field of human-robot interaction is experiencing significant growth as a field of scholarly endeavor [7], [8]. Through the Personal Rover Project, we have focused specifically on the application of interactive, physically embodied robotic technology to informal learning environments [5]. This agenda has been motivated by our and others' results which show that educational robotics can trigger significant learning that extends well beyond STEM (science, technology, engineering and mathematics) and into associated lifelong skills of problem-solving and communication [1], [4], [5], [9], [11], [12], [13], [14], [16], [17], [18], [19].

Prior robot design efforts from the Personal Rover Project lead to the creation and use of the Trikebot educational robot [10]. As a tool for intensive curriculumbased use, the Trikebot achieved its educational goals by maximizing mechanical transparency, using a slotted construction technique to enable complete construction and repair by students over eight weeks of intensive use.

In contrast to the Trikebot robot, the present project demanded a robot that could be subjected to tens of thousands of interactions without failure. Mechanical transparency would be secondary to design aesthetics,

\footnotetext{
* This work was funded by NASA/Ames and Intel Corporation.
}

interface transparency and robustness to naive use. Dubbed the Personal Exploration Rover (PER), our resulting interactive science rover is meant for prolonged use in unmediated settings, by novice users, without suffering from the fragility and susceptibility to failure often seen in interactive robotics devices. The PER has been designed as a robotic introduction to the technologies that enable NASA's missions and as an immersive, educational tool for experiencing the challenges faced by NASA mission scientists. PER project objectives are:

- Demonstrate that rovers are tools for doing science by enabling visitors to act as mission scientists, using the PER to conduct a science operation.

- Enable visitors to appreciate the role of autonomy in robotic systems, in particular planetary exploration rovers.

The PER installations present museum visitors with the challenge of searching for signs of life on rocks in a physical Marscape simulation or Mars yard. Using a carefully designed kiosk-based user interface to communicate with the rover, visitors interpret panoramic imagery and orthographic, overhead imagery to identify a science target of interest, then monitor as the PER approaches the rock, scans to find the target's exact position, corrects and maneuvers autonomously for a close approach, then conducts an ersatz test for signs of life.

Significant research results span physical robot design, robot software architecture and human-robot interaction design. Key enabling advances include the areas of power management, terrain inference, science target approach, and software architecture. In just the first two months of operation, PERs effected more than 20,000 autonomous science target approaches, achieving Mean Time Between Failure performance exceeding one week of use. Greater than 30 miles of rover travel were completed with idle times approaching $0 \%$ of museum operating hours at the Exploratorium. There were only nine robotic failures in this time span, all of which were straightforward servomotor failures, easily repaired by the replacement of a hobby servo.

To date, PER robot installations have operated at five national museums, including the Smithsonian National Air \& Space Museum (NASM) and the San Francisco Exploratorium. Our results suggest that the time for reliable, robotic engagements in public spaces is here. This paper describes rover mechanical design, embedded 
electronics architecture, software architecture, and human-robot interaction design.

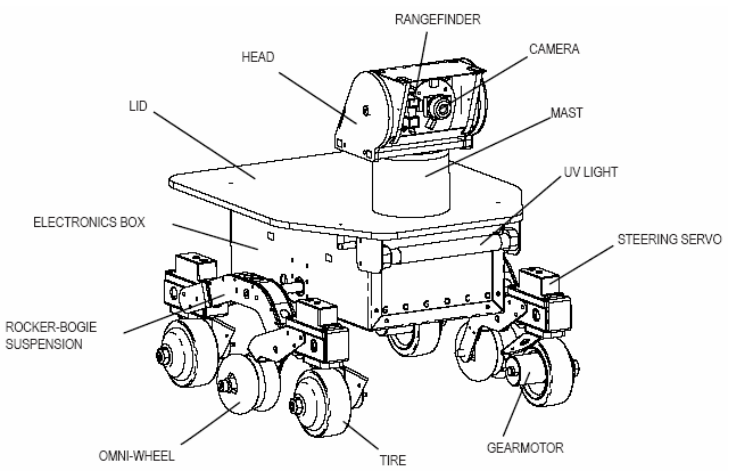

Fig. 1. PER chassis

\section{ROBOT DESIGN}

\section{A. Mechanical summary}

Physical Description. The mechanical chassis of the PER, designed by Gogoco in collaboration with Carnegie Mellon University, (Fig. 1) emulates the configuration of NASA's two Mars Exploration Rover (MER) robots currently exploring Mars. Like a MER, a PER has a six wheeled rocker-bogie suspension supporting a rectilinear body/electronics box. Above the electronics box is a camera head atop a short mast. Overall, the height of the PER is approximately $36 \mathrm{~cm}$, the length is $33 \mathrm{~cm}$ and the width is $34 \mathrm{~cm}$ (Fig. 2). The approximate weight, fully loaded, is $15 \mathrm{lbs}$.

Objectives and construction. First and foremost, the PER is a mobile camera platform. Like the MER, the PER needs to be able to traverse unstructured terrain while capturing images both for navigation and science study. Unlike the MER, the PER was designed to be relatively inexpensive so that many PERs could be built for multiple simultaneous exhibitions at an affordable price point, as with a previous Personal Rover Project robot, the Trikebot [9]. To this end the PER design process embraced the use of off-the-shelf electromechanical components whenever feasible (e.g. stock RC hobby type servos, batteries, gearmotors, etc.). Rather than designing the PER to have similar scale to the MER platforms, we chose to minimize the size of the PER, subject to off-the-shelf sensor, motor and microprocessor constraints, so that relatively small museum Mars yards would nevertheless yield rich interactions. The final size of the PER was ultimately determined by the size of the electronics box required to house the electronics boards (i.e. microprocessor board, motor controller, power board) and batteries.

The majority of custom-made parts are either laser cut plastic (Delrin) or formed sheet metal. Minimizing the number of machined parts minimizes parts costs while the anodized aluminum sheet metal construction helps to lend the PER a space hardware aesthetic.

Suspension and drivetrain. The PER rolls on six wheels using a rocker-bogie suspension system similar to that used by the MERs. There are three wheels in line on each side. The four corner wheels are powered by DC gearmotors and are independently steered by standard RC hobby servos through a total range of approximately 180 degrees. The tires are stock rubber, $6.8 \mathrm{~cm}$ diameter RC car tires mounted on custom hubs that allow the motors to be installed partially inside the wheel with the steering axis in line with the center of the wheel (Fig. 4). The two center wheels are omni-directional, freely spinning, Swedish 90 wheels. The overall wheelbase (distance between the center of the front and rear wheels) is $25 \mathrm{~cm}$, and the wheel width (distance between the center of the left and right wheel treads) is $26 \mathrm{~cm}$ (Fig. 2).

The rocker-bogie-type suspension was used in both the Sojourner rover and the recent MERs. Developed by JPL, it allows all six wheels to maintain full contact with an uneven surface without the use of springs or powered actuators (Fig. 3). It also averages out the displacement of all six wheels, minimizing the resulting tilt of the rover's main body as the suspension negotiates bumps and rocks. Such main chassis stability is particularly important to the MERs and PERs because it yields a more stable camera platform.

To minimize power and control requirements, only the corner wheels of PER are driven, unlike the MERs which have six driven and steered wheels. This is detrimental to the PER's ultimate ability to traverse tough terrain, but is adequate for the artificial Mars yard terrains and helps to mitigate cost and control complexity.

Having four independently steerable corner drivewheels along with omni-directional center wheels gives the PER great directional freedom. It can turn in place, translate sideways, or drive at an angle independent of orientation. While not technically holonomic because the pairs of motors on each leg always turn at the same speeds, this geometry enables both omnidirectional and Ackermannstyle motion.
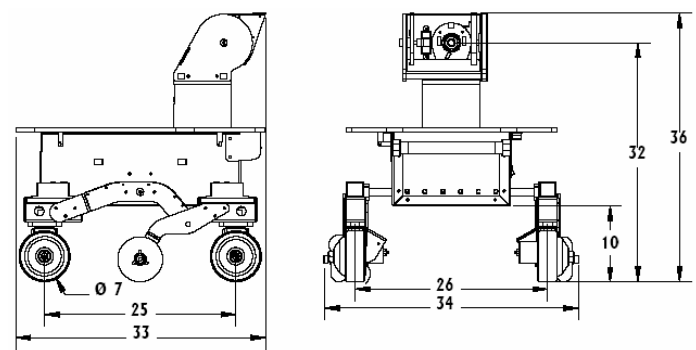

Fig. 2. PER front and side view dimensions in centimeters.

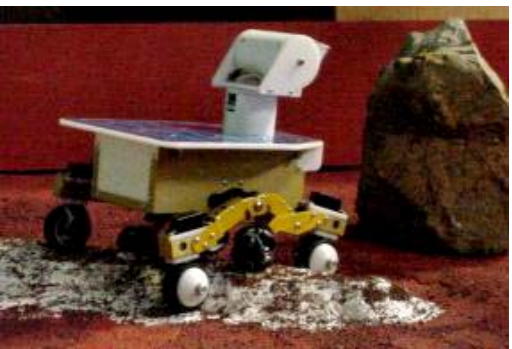

Fig. 3. The rocker-bogie suspension helps stabilize the PER and keep all six wheels on the ground as the PER traverses uneven terrain. 


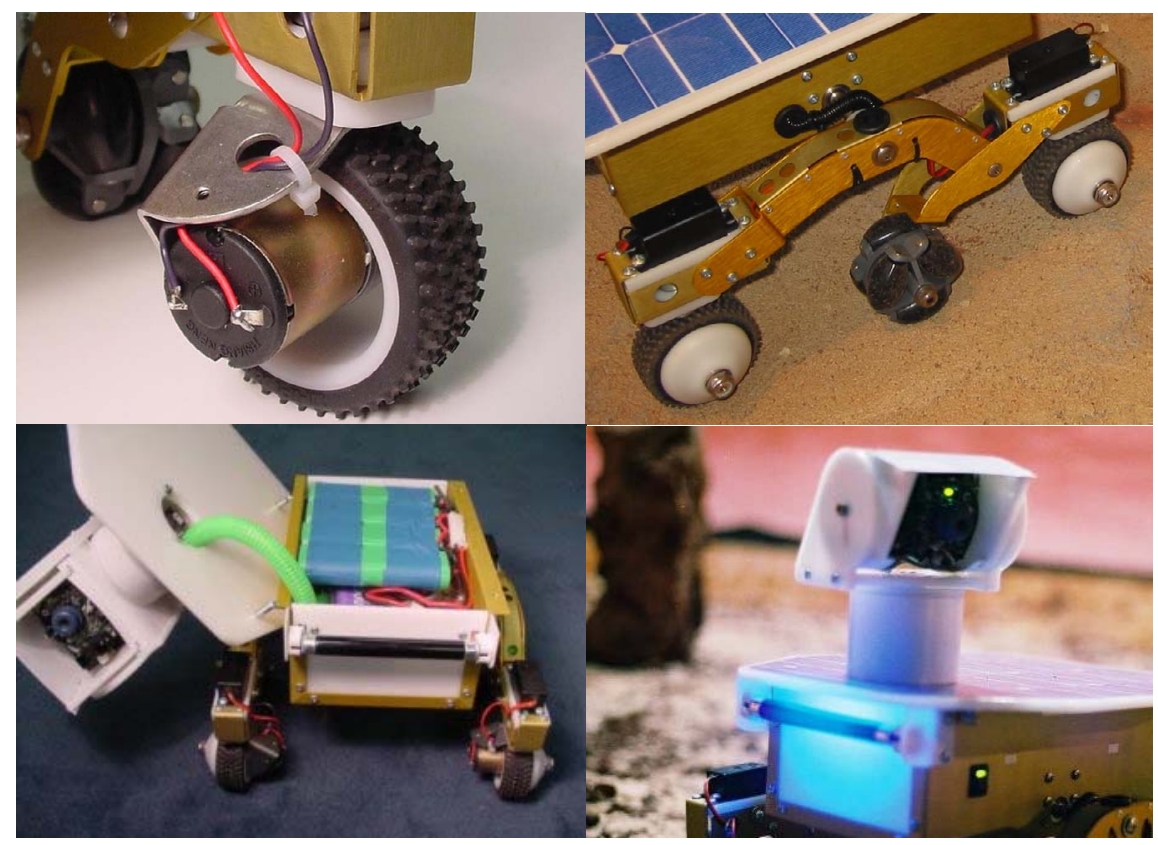

Fig. 4. (A) Custom wheel hubs allow the steering axis to be in line with the center of the wheel. (B) The rocker-bogie suspension and omni wheel. (C) The PER's lid swings open for easy access to the battery pack. (D) The pan-tilt head makes the PER's direction of focus clear to observers. The UV light on the front of the rover is used to analyze target rocks. Additional pictures can be found at http://www.cs.cmu.edu/ personalrover/PER/gallery.html.

Electronics box. The suspension system carries the electronics box where the batteries and electronics are mounted. The lid of this box is hinged to allow easy access to the batteries (Fig. 4). The power switch and a UV fluorescent light are also mounted to the exterior of this box. The box is fully enclosed to protect the electronics from dust and damage. For strength, the bottom and sides are metal while the ends and lid are made of plastic for radio transparency to allow the $\mathrm{WiFi}$ link in the electronics board to operate. The lid is shaped to be reminiscent of the "winged" solar panels on the MER.

Camera and camera mast. A camera and optical IR range-finder are mounted on the rover's pan and tilt head. The pan axis can rotate $\pm 180^{\circ}$ from center, allowing $360^{\circ}$ panoramas to be made with the camera. The tilt axis mechanical range is $+90^{\circ} /-45^{\circ}$ from horizontal. The camera lens axis intersects the pan axis to help simplify the interpretation of imagery; panning does not also cause translation of the point of view. Based on prior results regarding diagnostic transparency, great care was taken to design the PER's pan-tilt head so that it demonstrates a clear direction of attention (Fig. 4). The motion of the head as it pans and tilts to search for obstacles and science targets facilitates an understanding by museum visitors regarding the level of attention PER pays to its surroundings.

\section{B. Electronics}

Processors. Fig. 5 shows a schematic of the PER's electronic system. For low-level control of motors and for reading sensors we use a Cerebellum control board. This PIC microprocessor-based board was designed by the Robotics Institute (Carnegie Mellon University) and
Botrics, LLC. It can command two pairs of DC motors and $8 \mathrm{R} / \mathrm{C}$ style servos. It can also read 8 analog inputs and additional digital inputs.

The PER's main processor, the Stayton board, is an embedded computer designed by Intel Corp. for robotics applications. This single-board computer runs the Linux operating system on a $400 \mathrm{MHz}$ ARM processor. Onboard memory consists of $32 \mathrm{MB}$ Flash ROM and $64 \mathrm{MB}$ RAM. This board communicates with the rover's camera over USB, the Cerebellum over RS232 serial running at 115200 baud, and via $802.11 \mathrm{~b}$ wireless Ethernet with the mission control interface running on a PC.

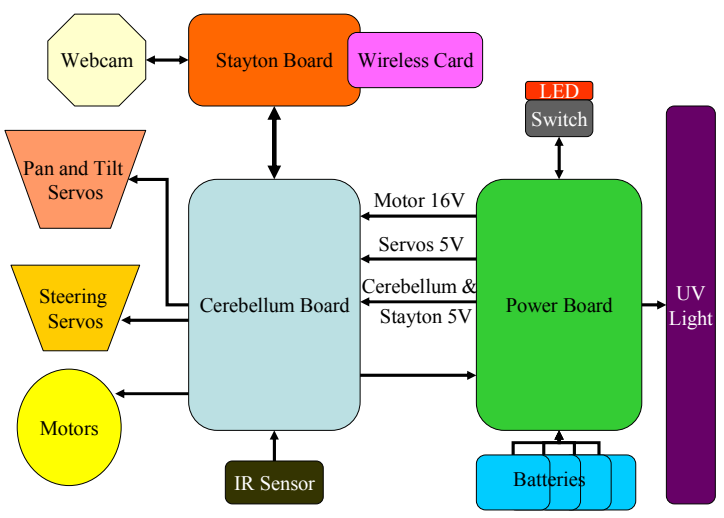

Fig. 5. PER electronics schematic.

Power. The rover is powered by four 7.2 volt nickel metal hydride batteries connected in series. Their total capacity is 3 Amp-hours at 28.8 volts. Botrics, LLC designed the power board exclusively for the PER. This board distributes power from the 28.8 volt battery pack to 
all the on-board electronic devices. Its total power output is $4 \mathrm{Amps}$ at 5 volts (to processors), 5 volts (to servos), and 16 volts (to motors). A fully charged pack powers the rover for about 10 hours during typical museum usage. Full-day battery life and overall rover endurance represented a critical performance target for the PER project due to demands placed on staff time at high-traffic science centers and museums. There simply is no time to replace batteries or charge batteries mid-day in such facilities. The solution demanded the use of low-power processors, including PIC microprocessors and an ARMbased main processor in lieu of a standard PC platform. A single high-voltage battery bus, fed via high-efficiency switching power supplies, designed with current capacities for each required voltage source, provided the second half of the answer. By eliminating power-hungry, cheap regulators on each processor board, we induced significant savings by simply avoiding the conversion of excess onboard energy to heat.

Output. The four rover drive motors have a 332:1 gear ratio that allows them to spin at a near constant velocity whether or not they are under load. The rover drives each motor at 16 volts giving the PER a top speed of 4 $\mathrm{cm} /$ second. Because the Cerebellum is only able to drive two independent motors, the motors on the left side use one channel and the motors on the right side use the other.

The PER's steering and head angles are all driven by hobby servos. The steering servos are low-profile, hightorque servos with brass shafts. The pan servo is designed for use in R/C sailboats as a winch and is capable of turning more than 360 degrees. An ultraviolet bulb and driver electronics enable the PER to simulate a test for signs of life. Target rocks painted with a fluorescent paint that is not visible under ordinary light shine blue under the UV light, indicating the simulated signs of life.

Sensors. The rover has a USB camera and an IR rangefinder mounted in its pan-tilt head. The camera, a Creative WebCam Pro, is used for both panoramic imaging and close-up target imaging. It has a maximum frame rate of 15 frames/second and maximum resolution of $640 \times 480$. An infrared triangulation-based rangefinder is used to find distances. The Sharp model 2Y0A02 rangefinder returns point distance readings accurate between $20 \mathrm{~cm}$ and $150 \mathrm{~cm}$. The rover uses this rangefinder to scan for obstacles in its path during traverses and to identify the exact distance and bearing to target rocks. A connection between the power board and the Cerebellum allows the rover to monitor battery voltage.

\section{Software architecture}

Cerebellum. The software on the Cerebellum is designed to be as simple as possible. Following that principle, it only accepts a single type of command that specifies the positions for the six servos, the motor velocities, and the state of the UV bulb. It always returns the same type of response containing a status byte along with the IR range and battery voltage. The Cerebellum limits the speed at which the servos move to put less stress on the motors. To ensure safe operation of the robot, if no valid command has been received in the last $120 \mathrm{~ms}$, the servos and drive motors are turned off.

Stayton. The Stayton only makes decisions that are too time-critical to be sent over a wireless link. When the robot is commanded to turn a specified number of degrees or drive a certain distance, the Stayton makes the decision as to when to stop the robot. While driving, the Stayton moves the head to scan for obstacles using the IR rangefinder and makes the decision to stop if an obstacle is detected.

While scanning for a rock, the rover sweeps the rangefinder through 270 degrees generating 91 range readings. In order to complete the scan quickly, the head cannot be allowed to come to a stop to record each distance. Complicating the action is the fact that the servos do not provide any position feedback. Every 50ms, the pan servo is commanded to a new position and the range reading is sent back. Through experimentation, it was determined that while scanning, the servo's actual position lags the commanded position by about $100 \mathrm{~ms}$. In this application, timing is critical to make sure that the scan readings are as accurate as possible; therefore, the scanning action is controlled on board the Stayton.

The other main function of the Stayton is to create an abstraction to a user controlling the robot. The drive motors all turn at slightly different speeds. The servo motors all have different center positions and different ranges of motion. For example, changing the pulse width from $1 \mathrm{~ms}$ to $2 \mathrm{~ms}$ may cause one servo to move 90 degrees and another to move 95 degrees. For this reason, every robot has a calibration file that is loaded when the Stayton program starts running. It tells the Stayton how to convert an angle into a servo position that the Cerebellum understands and stores the characteristics of the motors so that the rover can turn and drive accurately, despite inherent motor speeds that can vary as much as $25 \%$.

The Stayton keeps track of the positions of all six servo motors, which allows it to wait just the right amount of time for the servo motors to get to the commanded angle. This feature simplifies taking a picture so that the user can simply command that a picture be taken at a specific pan and tilt. The Stayton moves the head to that position and takes a picture when the head is in place. Requests for pictures can be queued up, so that right after a picture is taken, the head is moved to the position for the next picture before the image is compressed and sent. These features are used to create panoramic images without blur in as little time as possible.

Intel has licensed the Stayton technology to Crossbow Technology, Inc. The resulting Stargate board replaces the Stayton in subsequent builds of the PER with no noticeable differences.

PC. A java application running on a PC makes all decisions related to high-level mission execution. All navigation planning occurs on this level as well as the user mission interface. 


\section{Maintenance}

The PER robot is designed to be easily maintained. The parts most likely to fail, servos and motors, are available off-the-shelf and can be replaced without disassembling the entire rover. To assist museum staff in this maintenance, we created a manual that aids staff members in the diagnosis and repair of the PER. The manual covers simple diagnosis of problems, replacement of broken parts, and calibration of replacement parts via helper programs. A copy of the manual is available at www.cs.cmu.edu/ personalrover/PER/downloads.html.

Good documentation is most effective when coupled with diagnostically transparent behavior on the part of the robot, so that the museum staff can over time be empowered to, through observation and interaction, accurately identify the existence of and the source of failures in robot hardware or software. To this end a series of standalone interactive diagnostics are designed into the PER firmware, enabling the museum staff to test every degree of freedom and calibration point, without the need for a computer link to the PER, simply using gestures to communicate with the rover's rangefinder and camera. A second example of such diagnostic transparency involves low-battery warnings. In the case of low voltage, the PER commands the tilt angle of the head so that it is looking straight up. Upon familiarization with this behavior, museum staff found that they could confidently verify that all was well with the battery charge simply by glancing at the robot to ensure that it was not "looking up," a unique and unambiguous gesture from robot to human.

\section{INTERACTION DESIGN}

The final component of a robotic system for unmediated museum use is a user interface that allows novice users to easily control the robot and helps guide them through the desired interaction. In order to design such an interface, we assembled a team of designers, roboticists, and programmers. The team followed an iterative design process and used several methods to understand the scope, goals, and technical requirements of the project. An initial assessment of the existing interface and results from preliminary informal user tests revealed some areas for improvement. Specifically, three guiding goals for the interaction were set:

- Assure visitor throughput by enabling users to easily complete a mission in less than three minutes

- Communicate the idea that robots are tools for science and exploration.

- Demonstrate the rover's semi-autonomy-while a user gives the rover high-level commands, the rover makes smart decisions during mission execution.

Scenarios of use were essential tools in the design process and were created based on the informal user testing to illustrate a good and a bad experience with the rover exhibit from a user's perspective. Through rapid prototyping of the designs and successive cycles of informal user testing, the team was able to quickly eliminate problematic concepts and arrive at the following sampling of solutions.

Interface language. The prospective audience represents a broad range of scientific and technical expertise, so minimal formal scientific and technical terminology is used. Instead, a simple, game-like tone supports the interaction.

Interaction Cues. The default screen display, or "attract loop", on the kiosk provides a visual overview of the mission, foreshadowing steps the user might take. The kiosk itself has a simple track ball and a button set-up, similar to an arcade game. The mission begins when the user presses the button. A linear interaction follows as the mission unfolds step-by-step to the user.

Physical Orientation. To help the user orient between the Mars yard and the information displayed on screen, a Martian sun is painted on the far wall of the Mars yard and is visible from both the kiosk and in the panoramic view on screen (Fig. 6). In addition, the target rock positions, rock morphology, and the shape of the yard provide feedback and help users interpret the orthographic map imagery provided. An animation is used to help clarify the unfamiliar, 360-degree wrap-around vantage of the panoramic image.

Real-Time Feedback. A "Mission Builder" screen display (Fig. 7) tracks users' progress in real-time until they are ready to submit the mission to the rover. As the rover executes the mission, a rover's-eye view camera allows the visitor to experience the mission from the rover's perspective. The "Rover Mission" sub-window at bottom right remains visible during execution, providing data regarding rover operations, distance traveled and angles turned.

Visual Interface. A consistent color palette is used to unify the screens. Static and animated elements on the screen are designed to provide focal points for the users depending on the actions required. Consistent, clear typography provides visual hierarchy and improves readability [2].

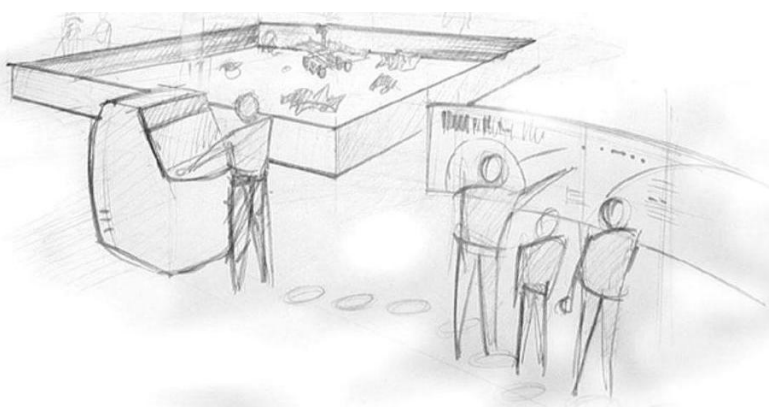

Fig. 6. The ability to see the yard and kiosk screen simultaneously aids users in orienting themselves within the exhibit. 


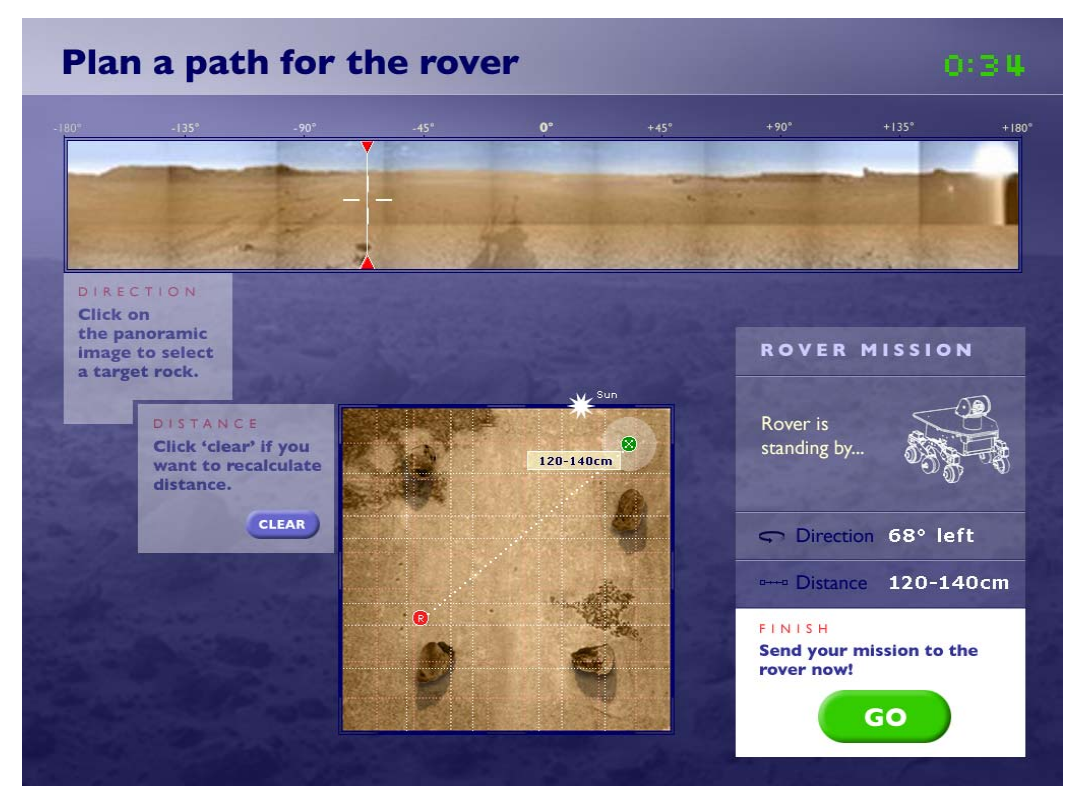

Fig. 7. The "Mission Builder" screen display. Graphic design by LotterShelly.

\section{ROVER PERFORMANCE}

The rovers have proven to be reliable, and, as seen from this museum staff comment, often more reliable than the museums had expected:

"Unlike most of our exhibits these [PERs] get slammed constantly, opening to closing, with no rest. I think they're holding up surprisingly well. Better than I thought they would."

Most failures are due to broken servos or motors, which are easily serviced by museum staff. When the exhibits opened, the first robot failures we found were in the tilt servos, drive motors, and steering servos. For all of these parts we made modifications to the robots. To mitigate drive motor failure, we reduced the motor duty cycle from $100 \%$ to $80 \%$ on Jan $12^{\text {th }}$. The steering servo speed was reduced also, and beginning Jan $27^{\text {th }}$, we modified the power board to send $5 \mathrm{~V}$ rather than $6 \mathrm{~V}$ to the servos. The original tilt servos had plastic gear teeth. Beginning Jan $27^{\text {th }}$, we replaced these servos with a metal-geared servo. Following these modifications, rover reliability was significantly improved (Fig. 8).

\begin{tabular}{|c|c|c|}
\hline & $\begin{array}{c}\text { Before Modifications } \\
\sim 38 \text { operating days/robot } \\
\text { for Tilt \& Steering } \\
\text { Servos } \\
\sim 13 \text { operating days/robot } \\
\text { for Motors }\end{array}$ & $\begin{array}{c}\text { After Modifications } \\
\sim 80 \text { operating days/robot } \\
\text { for Tilt \& Steering } \\
\text { Servos } \\
\sim 105 \text { operating } \\
\text { days/robot for Motors }\end{array}$ \\
\hline $\begin{array}{c}\text { Tilt } \\
\text { Servos }\end{array}$ & 6 & 4 \\
\hline $\begin{array}{c}\text { Steering } \\
\text { Servos }\end{array}$ & 8 & 16 \\
\hline Motors & 5 & 6 \\
\hline
\end{tabular}

Fig. 8. Number of broken rover components before and after rover modifications were implemented.
Although the robots have been in operation roughly 8 times longer after the motor modification than before, the total number of motor failures after modification is only slightly higher than the number of failures before modification.

The robots have been in operation roughly twice as long after the steering servo and tilt servo modifications as before the modifications. The number of tilt servo failures has decreased somewhat. The number of failed steering servos has doubled, as would be expected. However 13 out of the 16 post-modification servos that failed were located at a single site, the National Science Center. We believe that the high proportion of servo failures at that location is due in part to the rough surfaces of the National Science Center yards. Beginning June $1^{\text {st }}$, a new and more robust steering servo will be substituted as the old steering servos break.

The other rover components have proven to be quite reliable. In the roughly five months between Dec $29^{\text {th }}$ and June $1^{\text {st }}$, a single pan servo was broken due to an accident, four IR wires and three camera wires broke but were easily repaired, one camera broke, and one robot developed communication problems.

Robots ran an average of 8 days between failures before modifications and an average of 19 days between failures after the modifications. Looking at the total operating time between Dec $29^{\text {th }}$ and June $1^{\text {st }}$, robots ran on average 15 days before failure.

\section{EXHIBIT USEABILITY}

The Learning Research \& Development Center (LRDC) and the Institute for Learning Innovation (ILI) conducted formal educational evaluation of the PER exhibit. The analysis showed that the exhibit was successful in its goals of helping visitors explore the use of robots in science and the role of robot autonomy. The details of this analysis can be found in [15]. 
Quantitative statistics regarding exhibit use were collected automatically at installations by the exhibit software. The total number of robot-visitor interaction episodes exceeded 50,000 within the first nine months of operation and continues to grow. Significantly, the statistics show that time on task is extremely close to the design target of 3 minutes, well exceeding the 1.4 minute engagement time typically seen at interactive science exhibits [3]. More importantly virtually all exhibit users $(98 \%)$ were able to successfully design a mission and send it to the rover [15]. Together these statistics indicate that the distribution of time on task is not, as is often the case in museum exhibits, exponential but rather unimodal and narrow. Users who are engaged by the PER exhibit remain engaged through mission completion, then helpfully release control to the next museum visitor in queue.

About half of the missions conducted (52.7\%) ended with the rover successfully locating a rock. In $43.9 \%$ of the missions, the rover did not locate the target rock. The main causes for failing to find the target were that the rover reached a hip wall or the rover's path was blocked by a rock. The rover considers a rock to be an obstacle rather than the target when there are still more than 150 $\mathrm{cm}$ left in the mission. In these cases the user was given the option to try again. Only $1.9 \%$ of missions timed out at this stage, showing that users were highly engaged even when their mission failed to find the target rock. The mission ended due to a robot error such as failed communication in only $3.4 \%$ of the missions.

In summary it is clear both from time on task values, time-out rarity and mission success rates that visitors are able to effectively make use of the PER exhibit, even in the unmediated cases of the Exploratorium and NASA/Ames installations.

\section{CONCLUSIONS}

The Personal Exploration Rover has served as a rewarding demonstration of educational robotics applied to the informal learning space. This project demonstrates that robotic technology has compelling value in the museum setting, and that concrete educational results can be achieved and measured in such a setting. More than 40 PER's have been fabricated to date, with a mean time between failure exceeding two weeks for full-time usage by non-roboticists. Museum staff and docents have proven to be capable early adopters of mobile robot technology when presented with off-the-shelf replacement procedures that are well documented. Exhibit statistics show that virtually all users succeed in the completion of an entire scientific rover mission. Educational evaluation suggests that the exhibit effectively serves as a platform for family discussions about the MER mission and robotics, and that children come away from the exhibit with measurable knowledge in these areas.

As robotic technology advances, such interdisciplinary teams of engineers, interaction designers and education specialists will be capable of inventing and executing ever more compelling exhibits and curricula for both formal and informal learning venues. We hope that this project can serve as a motivation for future teams to not only research, dream and invent, but also to design, harden, fabricate and install so that thousands can benefit from these educational technology ventures.

\section{ACKNOWLEDGMENT}

We would like to thank all those at the museums who helped bring the PER to the public. We would also like to thank the following people for their contributions and support: Debra Bernstein, Jim Butler, Dan Clancy, Kevin Crowley, Maylene Duenas, Ed Epp, Rachel Gockley, Jean Harpley, Marti Louw, Anuja Parikh, Kristen Stubbs, and Peter Zhang.

\section{REFERENCES}

[1] Beer, R., Chiel, H., Drushel, R. Using autonomous robots to teach science and engineering. Communications of the ACM, June (1999).

[2] Carter, R. Digital Color and Type. Rotovision SA Hove, East Sussex, UK, (2002).

[3] Crowley, K., Callanan, M., Tenenbaum, H. \& Allen, E. (2001). Parents explain more often to boys than to girls during shared scientific thinking. Psychological Science, 12(3), 258-261.

[4] Druin, A. and Hendler, J. Robots for kids: exploring new technologies for learning, The Morgan Kaufmann Series in Interactive Technologies, Morgan Kaufmann, (2000).

[5] Ebert-Uphoff, I. Introducing parallel manipulators through laboratory experiments. IEEE Robotics \& Automation Magazine, 10 (3), pp. 13-19, (2003).

[6] Falcone, E., Gockley, R., Porter, E. and Nourbakhsh, I, The personal rover project, Special Issue on Socially Interactive Robots, Robotics and Autonomous Systems, (2003).

[7] Fong, T., Nourbakhsh, I. and Dautenhahn, K.. A survey of socially interactive robots. Robotics and Autonomous Systems, special issue, Socially Interactive Robots. 42 (3-4), pp. 143-166 (2003).

[8] Fong, T., Nourbakhsh, I. and Dautenhahn, K. A survey of socially interactive robots: Concepts, Design and Applications. Technical Report CMU-RI-TR-02-29, Carnegie Mellon University, Pittsburgh, PA USA, December (2002).

[9] Gerovich, O., Goldber, R. P., and Donn, I. D. From science projects to the engineering bench. IEEE Robotics \& Automation Magazine, 10 (3), pp. 9-12, (2003).

[10] Hsiu, T., Richards, S., Bhave, A., Perez-Bergquist, A. and Nourbakhsh, I. Designing a Low-cost, Expressive Educational Robot. In Proceedings of IROS 2003. Las Vegas, USA, (2003).

[11] Kitts, C. Surf, turf, and above the Earth. IEEE Robotics \& Automation Magazine, 10 (3), pp. 30-36, (2003).

[12] Kumar, D. \& Meeden, L. A robot laboratory for teaching artificial intelligence. In Proc. of $29^{\text {th }}$ SIGCSE Symposium on Computer Science Education, (1998).

[13] Murphy, R. Introduction to AI Robotics. MIT Press, (2000).

[14] Nourbakhsh, I., Crowley, K., Bhave, A., Hamner, E., Hsiu, T., Perez-Bergquist, A., Richards, S., Wilkonson, K. The Robotic Autonomy Mobile Robotics Course: Robot design, curriculum design and educational assessment. Autonomous Robotics Journal, in print. (2004).

[15] Nourbakhsh, I., Hamner, E., Bernstein, D., Crowley, K., Porter, E., Hsiu, T., Dunlavey, B., Ayoob, E., Lotter, M., Shelly, S., Parikh, A., Clancy, D. The Personal Exploration Rover: The Ground-up Design, Deployment and Educational Evaluation of an Educational Robot for Unmediated Informal Learning Sites. Carnegie Mellon University Technical Report CMU-RI-TR-04-38. August, (2004).

[16] Nourbakhsh, I. 2000a. When students meet robots. Essay in IEEE Intelligent Systems and Their Applications, 15(6), p. 15. (2000).

[17] Nourbakhsh, I. Robotics and education in the classroom and in the museum: On the study of robots, and robots for study. In Proceedings Workshop for Personal Robotics for Education. IEEE ICRA (2000).

[18] Papert, S. and Harel, I. Situating Constructionism, in: Constructionism, Ablex Publishing Corp., (1991).

[19] Wolz, U. Teaching design and project management with Lego RCX robots. In Proc. SIGCSE Conference (2000). 